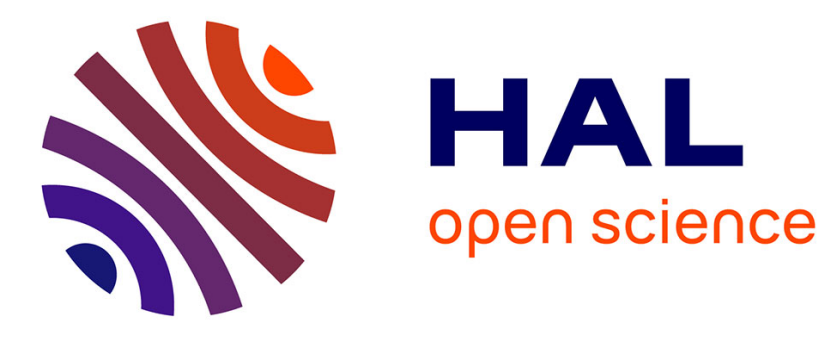

\title{
Avoir des enfants ?
}

Sandra Brée, Virginie de Luca Barrusse, Thierry Eggerickx, Anne-Françoise Praz

\section{To cite this version:}

Sandra Brée, Virginie de Luca Barrusse, Thierry Eggerickx, Anne-Françoise Praz. Avoir des enfants ?. Annales de démographie historique, 2016, 132 (2), 10.3917/adh.132.0005 . halshs-01624709

\section{HAL Id: halshs-01624709 https://shs.hal.science/halshs-01624709}

Submitted on 8 Jul 2021

HAL is a multi-disciplinary open access archive for the deposit and dissemination of scientific research documents, whether they are published or not. The documents may come from teaching and research institutions in France or abroad, or from public or private research centers.
L'archive ouverte pluridisciplinaire HAL, est destinée au dépôt et à la diffusion de documents scientifiques de niveau recherche, publiés ou non, émanant des établissements d'enseignement et de recherche français ou étrangers, des laboratoires publics ou privés. 


\title{
AVOIR DES ENFANTS? LES TRANSITIONS DE FÉCONDITÉ DEPUIS LA FIN DU XIX ${ }^{\mathrm{e}}$ SIÈCLE
}

\author{
par Sandra BRÉE, Virginie DE LUCA BARRUSSE, \\ Thierry EGGERICKX et Anne-Françoise PRAZ
}

L'évolution de la fécondité depuis la fin du XVIII -début du XIXe siècle et la recherche de ses déterminants ont fait l'objet de nombreuses recherches au cours des dernières décennies. Dans la foulée des monographies nationales et de la synthèse découlant du projet de Princeton, qui a notamment permis de dresser, avec précision et de manière comparative, la chronologie de la transition de la fécondité et de ses disparités spatiales en Europe, de nombreuses études se sont évertuées à tenter d'identifier, tant sur les plans théoriques qu'empiriques, les mécanismes explicatifs du changement radical des comportements reproducteurs. Pourquoi, dans des contextes socioéconomiques et culturels très différents, avec un synchronisme au demeurant assez relatif - le décalage entre les pays européens et en leur sein même se mesure en décennies - les populations ont-elles jeté aux oubliettes un régime démographique vieux de plusieurs siècles et ont-elles adopté de nouveaux comportements?

Ce changement s'inscrit dans un ensemble complexe et interactif de transformations de la société (urbanisation, industrialisation, sécularisation, développement de l'instruction, des démocraties, consumérisme...) et de chocs conjoncturels (crises économiques, guerres, épisodes épidémiques...) (Leboutte, 1995). Mais la simultanéité des transformations/changements ne suffit pas à produire un cadre explicatif satisfaisant et en tout cas généralisable. L'abondante littérature produite depuis plus de quatre décennies démontre qu'il existe un faisceau de raisons qui ont poussé les couples à subitement modifier leurs comportements de reproduction. Et ces causes, ces facteurs explicatifs, s'entremêlent et agissent avec des intensités variables selon les contextes. L'hétérogénéité qui en découle et qui s'observe au sein d'un même pays, d'une même région ou, à une échelle plus fine encore, souligne notamment l'importance des communautés locales et justifie pleinement les approches spatiales fines (Watkins, 1990). C'est ce que démontre une série de travaux récents comparant des communautés rurales et/ou des groupes sociaux; ils mettent en évidence l'éventail des réponses tant démographiques que socioéconomiques pour faire face aux tensions, aux contraintes et aux évolutions spécifiques (Alter et al., 2010, Debuisson, 2015, Matthys, Caron, 2014). Ces différentes stratégies permettent d'expliquer les décalages parfois importants dans la chronologie de la baisse de la fécondité, et d'identifier des groupes anticipateurs et des groupes résistants aux changements. 
Le terrain a donc été sérieusement défriché, mais de nombreuses zones d'ombre subsistent, en dépit de l'exploitation de bases de données de plus en plus riches et de méthodes d'analyses statistiques de plus en plus sophistiquées. Il importe, par exemple, de considérer le temps long de la transition et pas seulement la phase de déclenchement de la baisse décisive de la fécondité, et de replacer l'analyse de la fécondité "dans le processus de dynamique des populations, c'est-à-dire dans la chaîne des facteurs qui régissent l'évolution à long terme des effectifs de population" (Sardon, 1990, 947). C'est dans cette perspective de long terme que s'inscrivent certaines études récentes (Bengtsson, Dribe, 2014; Caldwell, 2006, 2008; Schellekens, Van Poppel, 2012). Par ailleurs, à l'instar de la plupart des articles de ce numéro des Annales de Démographie Historique, ce constat amène à considérer deux périodes moins étudiées : l'Entre-deux-guerres et celle du baby-boom (Kok, Van Bavel, 2010 ; Van Bavel, Reher, 2013).

L'Entre-deux-guerres marque, selon de nombreux auteurs, la fin de la première transition (Bengtsson, Dribe, 2014) car dans de nombreux pays, l'indice conjoncturel de fécondité atteint, dans les années 1930, des niveaux très bas, largement inférieurs au seuil de remplacement des générations (fig. 1). Comment expliquer cette très faible fécondité? S'agit-il du simple prolongement et de l'aboutissement du mouvement amorcé des décennies auparavant dans un contexte de déchristianisation, d'individualisme, de consumérisme de plus en plus prégnant (Van Bavel, 2009)? Les courbes de la figure 1 , abstraction faite de l'épisode tragique de la Première Guerre mondiale et de la courte phase de récupération des naissances qui la suit, apportent du crédit à cette hypothèse. Mais rien ne permet non plus d'exclure l'influence des forces conjoncturelles que furent la Grande dépression économique des années 1930 et ses conséquences sociales, ou encore de l'instabilité politique liée aux régimes totalitaires. Car après tout, ce contexte de crise a pu s'ajouter au cadre structurel évoqué plus haut pour amener la fécondité à un niveau très bas et retarder une reprise qui n'interviendra que vers la fin des années 1930, voire durant le second conflit mondial (Caldwell, 2008). Par ailleurs, si la plupart des pays occidentaux ont convergé vers ces basses fécondités, qu'en est-il des situations régionales ou sousrégionales? Certaines ont-elles échappé à cette lame de fond... et pourquoi? La plupart des études s'appuient sur l'analyse des indicateurs d'intensité de la fécondité - indice conjoncturel de fécondité ou descendance moyenne. Mais, que sait-on des mécanismes sousjacents, des stratégies élaborées pour restreindre la fécondité? Sont-elles identiques pour toutes les classes sociales? Dans le volume 4 de l'Histoire de la population française, G. Desplanques et J.-C. Chesnais (1988) soulignaient que les différences entre classes sociales demeuraient importantes: les classes moyennes et élevées étaient les moins fécondes alors que les plus pauvres assuraient le renouvellement des générations. Les réactions face à la crise semblent très différentes selon le groupe social. Compte tenu de l'importance de la stabilité d'un emploi et de son niveau de rémunération pour fonder une famille dans un contexte de récession économique, les hommes peu instruits et peu qualifiés font face à une difficulté accrue pour trouver une partenaire stable, ce 
qui se traduit par une diminution sensible des premières naissances. Chez les femmes instruites, la crise semble entraîner des stratégies de report des naissances alors que, pour les moins éduquées, la propension à avoir une première naissance serait stable ou augmenterait (Sobotka et al., 2011). Au-delà de quelques études, on ne peut que constater, pour cette période au moins, la rareté des études de démographie sociale et spatiale explorant la diversité des comportements et des mécanismes de contrôle ou non de la fécondité. Une partie de l'explication tient à la pauvreté des indicateurs disponibles au niveau agrégé, lesquels se résument le plus souvent aux taux de natalité, aux indices conjoncturels de fécondité et descendances moyennes. En l'absence d'information sur le calendrier de la fécondité, sur les intervalles entre naissances, sur les parités atteintes, ou encore sur la fécondité des femmes mariées et non mariées, on occulte l'analyse des mécanismes et des éventuelles stratégies d'ajustement (Sobotka et al., 2011; Bengtsson, Dribe, 2014). L'exploitation des données rétrospectives et individuelles extraites des recensements et des enquêtes, et auxquelles plusieurs articles de ce numéro ont recours, ouvre des perspectives nouvelles: par exemple, la production d'indicateurs de calendrier et de parités atteintes et leur croisement avec une variable sociale comme le niveau d'instruction, la seule qui présente une stabilité au cours de la vie des individus. Ces données, a priori confrontées à des biais de sélection liés aux sorties d'observation par décès et par émigration ainsi qu'aux non-réponses, ont été largement validées pour l'étude de la fécondité (Andersson et al., 2013; Brée et al., 2016a).
D'autres questionnements, relatifs à cette période, ont reçu peu de réponses à ce jour. C'est le cas par exemple du décalage entre les mesures natalistes prises dans de nombreux pays au lendemain de la Première Guerre mondiale et certains comportements individuels, résolument antinatalistes, tels que le refus de l'enfant pour bon nombre de couples ou le modèle de l'enfant unique (Desplanques, Chesnais, 1988). Pour les générations de femmes nées à la charnière des $\mathrm{XIX}^{\mathrm{e}}$ et $\mathrm{XX}^{\mathrm{e}}$ siècles, $40 \%$ des Françaises et des Anglaises ayant été mariées auront au mieux un enfant au terme de leur vie génésique. En Belgique, cette proportion atteint près de $50 \%$ (Brée et al., 2016b). La deuxième période, couverte par plusieurs articles de ce numéro, est celle du baby-boom. Globalement, l'explication des causes du baby-boom et l'identification des mécanismes - hausse de la nuptialité associée ou non à une augmentation de la fécondité - restent déficientes ou partielles (Van Bavel, Reher, 2013). Le babyboom est très souvent associé à la fin de la Seconde Guerre mondiale, tant dans sa temporalité que dans sa causalité. Or, comme le démontrent les courbes de la figure 1 et les contributions à ce numéro, la reprise de la fécondité se manifeste avant ou pendant les années de guerre et elle concerne tant les pays belligérants que ceux peu ou pas touchés par le conflit, tels que la Suisse ou la Suède. La guerre a ralenti, voire stoppé la reprise, avec pour les pays impliqués, un report des naissances: alors que, pour ceux qui sont restés neutres, la période 19351945 se caractérise par une augmentation importante de la fécondité. Enfin, tous les pays européens n'ont pas connu de baby-boom: c'est notamment le cas des pays du sud de l'Europe ou encore de 
Fig. 1 Évolution à long terme de l'indice conjoncturel de fécondité
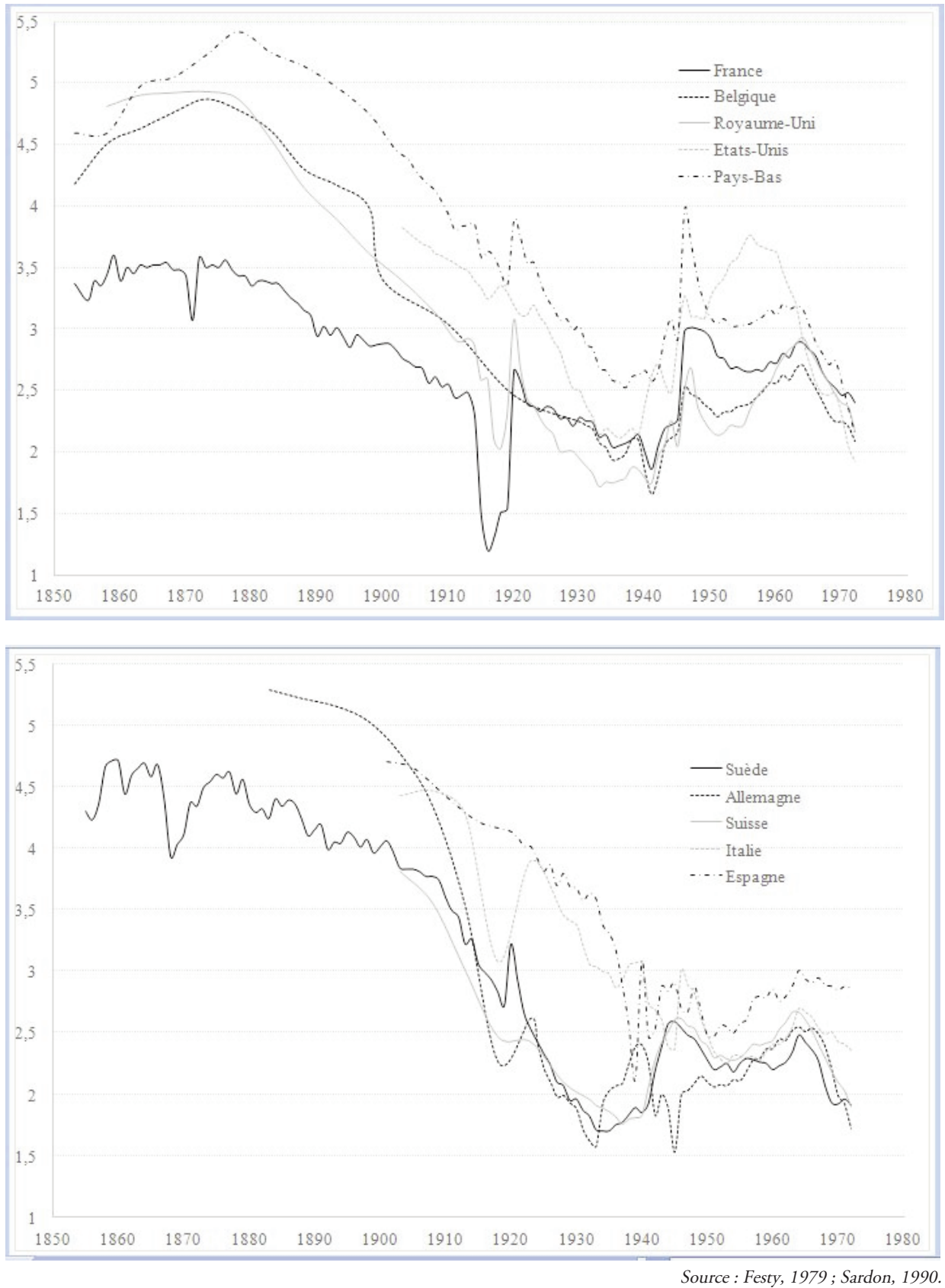

la Bulgarie et de la Hongrie. D’une manière générale, ce sont les pays où la fécondité était la plus basse avant-guerre

qui ont connu l'augmentation la plus rapide et importante (Van Bavel, Reher, 2013). 
Le baby-boom apparaît comme une rupture par rapport au malthusianisme d'avant-guerre, comme une véritable " révolution maternelle" (Knibiehler, 1997) valorisant la famille nombreuse, portée par les politiques familiales d'après-guerre et marquée par le modèle dominant de la mère au foyer et du père au travail. Cette période consacre également le retour au premier plan de la nuptialité; le mariage (re)devient la norme de l'union et se caractérise par sa précocité et sa durabilité (on divorce rarement).

Les articles présentés dans ce dossier éclairent ces zones d'ombre des transitions de fécondité. La contribution de Virginie De Luca Barrusse et Caroline Rusterholz synthétise des travaux qui empruntent parfois à plusieurs disciplines pour éclairer les motivations et les mécanismes des transitions de la fécondité. Elles se concentrent sur les études qualitatives couvrant la période 1850 1980 et montrent ce qu'elles doivent aux analyses quantitatives qu'elles viennent prolonger ou parfois contredire. L'approche en termes d'économie politique de la fécondité convoque les sciences sociales pour comprendre les ressorts des motivations de la régulation des naissances en interrogeant les niveaux macro et micro des contextes socioéconomique, politique et culturel dans lesquels se situent les prises de décisions des individus (Greenhalgh, 1995; Szreter, 2009, 2011; Szreter, Garrett, 2000). Ces recherches éclairent les motivations du changement du régime de la fécondité et montrent les ressorts des motivations individuelles à maitriser les naissances et leur calendrier. L'approche est prometteuse et mériterait d'être prolongée sans pour autant négliger les recherches quantitatives qui fournissent la mesure des décisions à l'instar de Duvoisin et alii. dans le présent numéro (Neven, Oris, 2003; Van Bavel, Kok, 2005; Schumacher 2010).

Les contextes économiques et sociaux ont retenu l'attention de plusieurs auteurs du dossier. C'est notamment le cas de l'article de Sandra Brée, Mélanie Bourguignon et Thierry Eggerickx consacré à la fécondité durant l'entredeux-guerres en Europe, une période caractérisée par de profonds bouleversements politiques, économiques et sociaux. Plus précisément, il s'agit, au travers d'une analyse de la littérature, tant théorique qu'empirique, d'explorer le lien entre les basses fécondités observées durant cette période et la crise économique des années 1930 et ses effets sociaux. Sur la base des indicateurs d'intensité les plus communément utilisés taux de natalité, indice conjoncturel de fécondité et descendance finale - les faibles fécondités atteintes dans les années 1930 (et par les générations de femmes nées au début du XXe siècle) se situent dans le prolongement du mouvement général amorcé au XIXe siècle. L'impact de la crise ne peut être certifié sur la base de ces seuls indices, mais il ne peut pas non plus être réfuté, car la détérioration du contexte socioéconomique a pu amener la fécondité à des niveaux anormalement bas et retarder la reprise, prémisse du baby-boom, qui n'interviendra qu'à la fin de la décennie, voire durant le second conflit mondial. L'exploitation des données rétrospectives des recensements et des enquêtes permet d'affiner l'analyse par la production d'indicateurs de calendrier de la fécondité ou de parités atteintes. Les quelques études recourant à ce type de données démontrent que les basses fécondités de l'Entredeux-guerres sont en grande partie liées à 
des niveaux élevés d'infécondité et à l'émergence du modèle familial de l'enfant unique. On observe aussi un report des naissances, et donc de l'âge à la maternité, une stratégie attentiste souvent utilisée en période de crise.

Hors d'Europe, l'article de Danielle Gauvreau et Benoît Laplante traite de la fécondité durant le baby-boom, avec une analyse comparative du processus dans les provinces de l'Ontario (protestant) et du Québec (catholique) examinant ainsi l'impact du facteur religieux. Le matériau utilisé est tiré d'informations rétrospectives recueillies lors du recensement de 1981; âge au premier mariage, nombre d'enfants mis au monde. La descendance finale des femmes, la variable dépendante, est mise en relation avec une série de variables indépendantes dont l'effet est estimé : religion, origine ethnique, niveau d'éducation de la femme, province de résidence (tenant compte de l'âge et de la durée du mariage). Les analyses, de statistique descriptive puis analytique, parviennent à départager le rôle du facteur nuptialité et du facteur fécondité dans l'augmentation générale de la fécondité du baby-boom. Les résultats montrent deux déroulements très différents de ce baby-boom. En Ontario, on retrouve le schéma classique où l'augmentation de la nuptialité et de la fécondité contribuent au baby-boom; ceci pour une durée très courte, puisque la courbe de fécondité repart ensuite à la baisse. Au Québec, l'évolution de la nuptialité explique en partie le babyboom.

L'effet des niveaux socioéconomiques et de l'éducation est testé dans dix communes italiennes entre la fin du XIX ${ }^{\mathrm{e}}$ siècle et le milieu du XX $\mathrm{XX}^{\mathrm{e}}$ par Marco Breschi, Massimo Esposito, Alessio
Fornasin et Matteo Manfredini. Grâce à des données individuelles de recensement, ils montrent que ces niveaux socioéconomiques et l'éducation des femmes sont les déterminants qui expliquent le mieux le calendrier de la transition de fécondité. Leur approche longitudinale rétrospective montre que, si les caractéristiques socioéconomiques expliquent bien les premières phases de la transition démographique, l'éducation des femmes a des effets sur l'ensemble des cohortes de leur corpus. Les femmes les plus instruites ont entamé leur transition de fécondité les premières, suivies, pendant l'Entre-deux-guerres, par celles n'ayant eu qu'une éducation primaire, puis après la Seconde Guerre mondiale, mais de façon encore marginale, par les femmes sans instruction. Ces femmes nées à partir du début du $\mathrm{XX}^{\mathrm{e}}$ siècle sont de plus en plus fréquemment éduquées, ce qui va de pair avec une plus grande indépendance et autonomie au sein de la famille (Cleland, 2001; Basu, 2002; Van de Putte, 2007). Pour les auteurs, la réduction des différentiels de fécondité, alors que l'éducation se propage dans la population féminine, est un élément compatible avec l'hypothèse de diffusion de baisse de la fécondité.

Eli Nomes et Jan Van Bavel se penchent également sur l'influence de l'éducation sur la fécondité. Ils adoptent une approche originale puisqu'ils étudient plus précisément la relation entre l'homogamie éducative et la fécondité pendant le baby-boom et le babybust. Les populations sont alors de plus en plus instruites et l'éducation devient un facteur toujours plus déterminant de la structure socioéconomique et un élément particulièrement important dans la sélection des partenaires (Breen, 2010). Pourtant, alors que le développement de 
l'instruction est souvent associé à une baisse de la fécondité, on observe l'inverse pendant le baby-boom (Ronsijn, 2014), au cours duquel le poids du niveau d'éducation sur la fécondité a baissé (Sandström, 2014; Van Bavel, 2014). Pour mieux comprendre ce phénomène, les auteurs n'étudient pas seulement les liens entre niveaux de fécondité (des hommes ou des femmes) et niveau d'éducation, mais tiennent également compte des combinaisons d'éducation des deux membres du couple pour saisir les implications sur la fécondité d'une plus forte homogamie éducative des époux. Leurs résultats montrent que les couples composés de deux partenaires peu instruits ont les plus forts niveaux de fécondité pendant le baby-boom alors que l'hypogamie (mari moins éduqué que sa femme) est associée à une plus faible fécondité. Les auteurs postulent d'ailleurs que l'augmentation de l'hypogamie pendant le baby-bust a pu être un facteur du déclin de la fécondité.

La fécondité en dehors de son cadre légitime mérite notre attention dans ce contexte de changement de comportement. C'est l'objet de l'article de Jan Kok et Hilde Bras. Les conceptions prénuptiales constituent un phénomène courant aux Pays-Bas pour la période 1870-1950. L'article explique les variations régionales de ce comportement grâce à plusieurs hypothèses. Le rôle des pratiques culturelles de fréquentations est avancé car elles peuvent entrâner une entrée précoce dans des comportements à risque, quoique le contrôle très serré du groupe de pairs diminue les risques de grossesse. L'hypothèse de l'homogamie est également mobilisée pour expliquer les conceptions prénuptiales car les parents «acceptent» les fréquentations dans un cercle de personnes connues.
Enfin, la volonté des jeunes de provoquer le mariage pour gagner leur indépendance peut être évoquée pour expliquer ces conceptions prénuptiales. Le matériau utilisé combine une base de données de quelques 10000 mariages, comportant des informations détaillées sur les époux, avec des informations sur les pratiques culturelles locales, issues d'une enquête ethnographique des années trente et d'un questionnaire rétrospectif de 1970. Ces données culturelles sont transformées en données chiffrées permettant de tester les différentes hypothèses. Il apparaît que les conceptions prénuptiales relèvent d'un comportement socialement marqué, mais aussi géographiquement déterminé. Il est plus fréquent chez les protestants que chez les catholiques.

Si l'étude de la fécondité amène à s'interroger sur le nombre d'enfants, les intervalles intergénésiques ou encore la taille des fratries, il convient de porter aussi son attention sur les femmes et les couples qui restent sans enfant, particulièrement dans un contexte de forte fécondité. C'est précisément l'objet du texte d'Aline Duvoisin, Sylvie Burgnard et Michel Oris qui s'intéressent aux contextes dans lesquels se déroulent les prises de décisions des individus qui restent sans enfant au cours du babyboom en Suisse. Il s'agit ici d'éclairer des comportements "marginaux» à un moment où, comme plusieurs travaux l'ont montré, la fécondité est fortement valorisée (Burgnard, 2015 ; Rusterholz, 2016). Alors que bien des études qui se sont focalisées sur le baby-boom ont eu recours à des données agrégées, les auteurs recourent à une analyse séquentielle de la trajectoire des personnes sans enfant grâce à des données originales et à des entretiens menés auprès d'un 
échantillon d'hommes et de femmes. Ils montrent l'hétérogénéité des situations mais aussi des logiques qui sont les leurs en matière de reproduction. Les auteurs mettent en lumière l'impact du niveau d'éducation des femmes sur leurs décisions, un niveau qui conditionne la fréquence et l'âge au mariage. Au final, l'article confirme les apports des approches qualitatives et quantitatives combinées et invitent à prolonger ce type d'analyse sur les marges des transitions démographiques, qu'il s'agisse des couples sans enfant dans d'autres pays mais aussi des familles nombreuses; en somme des comportements "atypiques» dont la singularité éclaire les normes en vigueur.

Si le présent dossier éclaire bien des aspects des transitions de fécondité depuis le XIXe siècle, il n'épuise pas la question. Il invite plutôt à prolonger les analyses pour comprendre tant les comportements majoritaires que minoritaires et ce qui les déterminent.

Sandra BRÉE

Centre de recherche en Démographie, Université catholique de Louvain sandra.bree@uclouvain.be

Virginie DE LUCA BARRUSSE

Université Paris-Panthéon Sorbonne virginie.barrusse@univ-paris1.fr

Thierry EGGERICKX Centre de recherche en Démographie, Université catholique de Louvain thierry.eggerickx@uclouvain.be

Anne-Françoise Praz

Université de Fribourg anne-francoise.praz@unifr.ch

\section{RÉFÉRENCES BIBLIOGRAPHIQUES}

Alter, Georges, Oris, Michel et Neven, Muriel (2010), «Le déclin de la fécondité dans les campagnes de Belgique orientale. Illustration d'une réponse multiphasique», 489-525, in Eggerickx, Thierry et Sanderson, Jean-Paul (dir.), Histoire de la population de la Belgique et de ses territoires, Chaire Quetelet 2005, Louvain-La-Neuve, Presses Universitaires de Louvain.

ANDERSSON Gunnar et SOBOLEv, Boris (2013), "Small effects of selective migration and selective survival in retrospective studies of fertility", Revue Européenne de Démographie, 29(3), 345-354.

BASU, Alaka M (2002), "Why education leads to lower fertility? A critical review of some of the possibilities", World Development, 30, 1779-1790.

Bengtsson, Tommy, Dribe, Martin (2014), "The historical fertility transition at the micro level: Southern Sweden 1815-1939 ", Demographic Research, vol. 30, 17, 491-534.

BréE, Sandra, EgGerickX, Thierry, SAnderson, Jean-Paul, Costa, Rafael (2016a), «Comparaison des données retrospectives de fécondité dans les recensements en Belgique et les enquêtes Famille en France», Population, 71(1), 85-120.

Brée, Sandra, EgGERICKX, Thierry, SANDERSON, Jean-Paul (2016b), "Low fertility, childlessness and family changes during the first half of the 20th century in France and in Belgium", Revue Quetelet, (à paraître).

BREEN, Richard (2010), "Educational Expansion and Social Mobility in the $20^{\text {th }}$ Century", Social Forces, 89(2), 365388. 
Burgnard, Sylvie (2015), Produire, diffuser et contester les savoirs sur le sexe. Une sociohistoire de la sexualité dans la Genève des années 1970, Berne, Peter Lang.

CALDWELl, John (2006), "The Western fertility decline: reflections from a chronological perspective", Journal of Population Research, 23, 2, 225-242.

CALDwell, John (2008), "Three fertility compromises and two transitions", Population Research Policy review, 27, 427446.

Cleland, John (2001), "The effects of improved survival on fertility: A Reassessment", Population and Development Review, 27, 60-92.

Debuisson Marc, (2015), Régimes démographiques et structures socioéconomiques. Les communautés villageoises de la province de Namur au cours de la première moitié du $19^{\mathrm{e}}$ siècle, Berne, Peter Lang.

Desplanques, Guy et Chesnais, JeanClaude (1988), "Les vicissitudes de la fécondité», 287-344, in Dupâquier, Jacques (dir.), Histoire de la population française, t. 4., De 1914 à nos jours, Paris, PUF.

Festy, Patrick (1979), « La fécondité des pays occidentaux de 1870 à 1970. Présentation d'un cahier de l'INED ", Paris, INED-PUF, 163-173.

Greenhalgh, Susan (1995), Situating Fertility: Anthropology and Demographic Inquiry, Cambridge, Cambridge University Press.

KnibieHLer, Yvonne (1997), La révolution maternelle depuis 1945, Paris, Perrin.

KoK, Jan et VAN BAVEL, Jan (dir.) (2010), De levenskracht der bevolking. Sociale en demografische kwesties in de Lage Landen tijdens het Interbellum, Leuven, Universitaire Press Leuven.

Leboutte, René (1995), "Les transitions démographiques dans les mutations globales des sociétés occidentales des $18^{\mathrm{e}}$ et $19^{\mathrm{e}}$ siècles", 9-32, in Tabutin Dominique, Eggerickx Thierry et Gourbin Catherine, Transitions démographiques et sociétés, Chaire Quetelet 1992, Louvain-la-Neuve, Institut de Démographie.

Matthys, Christa, Caron, Marianne, (2014), «La fécondité légitime dans différents agrosystèmes sociaux en Flandre, 1830-1930 », Espace, Populations, Sociétés, 2014/1, mis en ligne le 31 mai 2014, DOI: $10.4000 /$ eps.5671.

Neven, Muriel, Oris, Michel (2003), "Contrôle religieux, contrôle social: la fécondité dans l'est de la Belgique dans la seconde moitié du XIX siècle", Annales de Démographie Historique, 106, 5-32.

Ronsijn, Wouter (2014), "Educational Expansion and Gender Inequality in Belgium in the Twentieth Century", Histoire \& Mesure, 29(1), 195-218.

Rusterholz, Caroline (2016), "Deux enfants c'est déjà pas mal!" Famille et fécondité en Suisse, 1955-1970, Lausanne, Antipodes.

SANDSTRÖM, Glenn (2014), "The mid-twentieth century baby boom in Sweden changes in the educational gradient of fertility for women born 1915-1950", The History of the Family, 19(1), 120-140.

SARDON, Jean-Paul (1990), " Le remplacement des générations en Europe depuis le début du siècle ", Population, 6, 947-967.

Schellekens, Jona, Van Poppel, Frans (2012), "Marital fertility decline in the Netherlands: child mortality, real wages, and unemployment, 1860-1939”, Demography, 965-988.

SCHumaCHER, Reto (2010), Structures et comportements en transition: la reproduction démographique à Genève au $X I X^{e}$ siècle, Berne, Peter Lang.

Sobotka, Tomas, SkirbekK, Vegard, PhiliPOV Dimiter (2011), "Economic recession and fertility in the developed world", Population and Development Review, 37, 267-306. 
SZRETER, Simon (2009), “Questions, Questions, Questions! The Expanding universe of Resarch on Fertility Declines", Department of Geography, Downing College, University of Cambridge.

SZRETER, Simon (2011), "Theories and Heuristics: How to Approach the Study of Historic Fertility Declines?", Historical Social Research, 36, 65-98.

SzRETER, Simon, GARrETT, Eilidh (2000), "Reproduction, Compositional Demography, and Economic Growth: Family Planning in England Long before the Fertility Decline", Population and Development Review, 26, 45-80.

VAN BAVEL, Jan (2009), "Subreplacement fertility in the West before the baby boom: past and current perspectives", Population Studies, 64,1,1-18.

VAN BAVEL, Jan (2014), "The mid-twentieth century baby boom and the changing educational gradient in Belgian cohort fertility", Demographic Research, 30, 1, 925-962.
VAn Bavel, Jan, KoK, Jan (2005), “The role of religion in the Dutch fertility transition: Starting, spacing, and stopping in the heart of the Netherlands, 18451945", Continuity and Change, 20, 2, 247-263.

Van Bavel, Jan, Reher, David (2013), "The baby boom and its causes: what we know and what we need to know", Population and Development Review, 39, 2, 257-288.

VAN de PUTTE, Bart (2007), “The influence of modern city life on marriage in Ghent at the turn of the twentieth century: Cultural struggle and social differentiation in demographic behavior", Journal of Family History, 32, 433-458.

Watkins, Susan Cotts (1990), "From local to national communities: the transformation of demographic regimes in Western Europe, 1870-1960", Population and Development Review, 16, 2, 241-272. 